\title{
A Case of Mistaken Identity: Juvenile Myoclonic Epilepsy Manifesting as a Tic Disorder
}

\author{
Okeanis Vaou and Anna DePold Hohler*
}

Boston Medical Center, Boston University School of Medicine, Boston, MA, USA

\begin{abstract}
We describe a 20 year old female who presented to our movement disorders clinic for involuntary twitching of her neck and eyes for the past three months. The twitching occurred in clusters, without diurnal variation, lasting from a few seconds to five minutes. Her neurological exam was unremarkable except for mild slowing of processing speed and a childlike affect. Our differential included tic or seizure disorder. Her biochemical work up and brain MRI were normal. A routine electroencephalogram (EEG) was performed which revealed multiple episodes of paroxysmal generalized rhythmic sharp 3-4Hz activity, findings consistent with a juvenile myoclonic epilepsy (JME) variant. She was treated with levetiracetam and progressively titrated up to $1500 \mathrm{mg}$ daily with resolution of the electrographic discharges along with the clinical events. We believe that an EEG should be considered in the evaluation of atypical tic disorders, as shown in this unusual presentation of JME.
\end{abstract}

Keywords: Tics; Twitching; Involuntary movements; Juvenile myoclonic epilepsy

\section{Introduction}

Tics are characterized by abrupt, repetitive, stereotyped and involuntary movements (motor tics) or sounds (phonic tics). They are commonly preceded by a premonitory sensation of an urge, tension and discomfort, or other sensory phenomena $[1,2]$ The majority of tic disorders are genetic or idiopathic in nature with a usual age of onset in childhood [3]. For that reason, tics presenting in adulthood are most likely due to secondary causes.

Phenomenologically tics are classified as motor and vocal, or simple and complex. Simple motor tics are single repetitive jerks. Complex motor tics involve a sequential pattern of individual tics or more complex, coordinated actions that can resemble purposeful movements. Simple motor tics may need to be differentiated from stereotypies which seem to be longer lasting. They also need to be differentiated from myoclonic jerks, which are not typically repetitive in the same body part like tics, as we present in our patient.

\section{Case Report}

We present a 20 year old right handed female who presented for assessment of involuntary movements of her neck, shoulder and eyes.

A few hours following a trivial, uneventful motor vehicle accident, the patient noticed sudden jerky movements of her neck and shoulders. These movements occasionally coincided with involuntary movements of her eyes, looking up to either the upper left or right and would last from a few seconds to no more than one minute and occur multiple times randomly in the day. No alteration of consciousness was reported during or after these episodes, the patient was cognizant of these movements and could rarely suppress them. Stress did not worsen these episodes, an urge before these jerky movements was absent and no warning symptoms occurred before each episode. The patient denied similar movements in the past or in other parts of her body. Her past medical history was unremarkable. Family history of tic disorders, involuntary movements or seizures was absent. She denied toxic habits and was on birth control bills.

Neurological examination was unremarkable except for a notable child-like affect. No involuntary movements or twitching was noted. Clinical investigation including thyroid function and ASO antibodies were normal. A head MRI revealed benign capillary telangiectasia of the left pons. A routine EEG was consistent with an anterior background of beta and theta activity with multiple episodes of paroxysmal generalized rhythmic $3-4 \mathrm{~Hz}$ of sharp activity.

The patient was diagnosed with juvenile myoclonic epilepsy variant and treated with leviteracetam $1500 \mathrm{mg}$ daily. The episodes of involuntary jerky movements decreased in frequency and intensity and the repeat EEG showed decreased activity without accompanying clinical manifestations.

\section{Discussion}

Juvenile Myoclonic Epilepsy (JME) is idiopathic, hereditary generalized epilepsy consisting of irregular myoclonic jerks, generalized tonic clonic seizures or absence seizures which are not accompanied by loss of consciousness. Myoclonic movements, mostly of the arms, occur shortly after awakening. These are often preceded by a few minutes by generalized tonic-clonic seizures. Seizures are commonly precipitated by sleep deprivation, fatigue and alcohol consumption. The age of onset for the seizures is typically in adolescence and misdiagnosis and delayed diagnosis is common [4,5] Only 3-6\% of JME patients have only myoclonic jerks [6]. Generalized tonic clonic seizures (GTCS) appear years after onset of myoclonias [7].

Patients with JME have a normal background in electroencephalogram (EEG). Interictal findings include bursts of generalized and irregular spike-and-waves and polyspike-and- waves, in a frequency of 3-5 Hz with frontocentral dominance [8]. The typical ictal EEG of a myoclonic seizure manifests as a pattern of polyspikeand-wave, which consists of a group of 5 to 20 generalized, almost

*Corresponding author: Anna DePold Hohler, Boston Medical Center, Boston University School of Medicine, Boston, MA 02118, USA Tel: 516-851-3872; Fax: 617-638-8465, E-mail: anna.hohler@bmc.org

Received March 06, 2012; Accepted March 07, 2012; Published March 12, 2012

Citation: Vaou O, Hohler AD (2012) A Case of Mistaken Identity: Juvenile Myoclonic Epilepsy Manifesting as a Tic Disorder. J Neurol Neurophysiol 3:125. doi:10.4172/2155-9562.1000125

Copyright: ( 2012 Vaou O, et al. This is an open-access article distributed under the terms of the Creative Commons Attribution License, which permits unrestricted use, distribution, and reproduction in any medium, provided the original author and source are credited. 
Citation: Vaou O, Hohler AD (2012) A Case of Mistaken Identity: Juvenile Myoclonic Epilepsy Manifesting as a Tic Disorder. J Neurol Neurophysiol 3:125. doi:10.4172/2155-9562.1000125

Page 2 of 2

always symmetrical and high frequency $(10-16 \mathrm{~Hz})$ spikes, usually followed by slow waves in a frequency of $2,5-5 \mathrm{~Hz}$ [8]. As in our patient, the events of myoclonic jerks occur simultaneously with the polyspikeand-wave pattern and the latter may persist after the cessation of the myoclonus for several seconds.

JME requires lifelong treatment since seizures recur if the antiepileptic medications are discontinued [7]. First line treatment is Valproic acid and Lamotrigine. Levetiracetam, Clonazepam and Topiramate may be used as a second line agent. Our patient was placed on Levetiracetam at $750 \mathrm{mg}$ twice daily and by her report, she had significant improvement of her myoclonic jerks. A follow up EEG showed an absence of polyspike waves with some infrequent interictal sharp waves. There was no clinical correlation with the electrical discharges at that point.

Even though JME is the most common idiopathic generalized epilepsy [9], it is often missed or misdiagnosed. Myoclonic movements may typically precede the onset of GTC seizures and they may be mistaken for tics or myoclonic disorders, as in our case. A comprehensive history and a routine or prolonged EEG monitoring is warranted to reach the diagnosis of JME.

\section{References}

1. Jankovic J (2002) Tourette's syndrome. N Engl J Med 345: 1184-1192.

2. Leckman JF, Peterson BS, King RA, Scahill L, Cohen DJ (2001) Phenomenology of tics and natural history of tic disorders. Adv Neurol 85: 1-14.

3. Chouinard S, Ford B (2000) Adult onset tic disorders. J Neurol Neurosurg Psychiatry 68: 738-743.

4. Atakli D, Sözüer D, Atay T, Baybas S, Arpaci B (1998) Misdiagnosis and treatment in juvenile myoclonic epilepsy. Seizure 7: 63-66.

5. Grünewald RA, Chroni E, Panayiotopoulos CP (1992) Delayed diagnosis of juvenile myoclonic epilepsy. J Neurol Neurosurg Psychiatry 55: 497-499.

6. Renganathan R, Delanty N (2003) Juvenile myoclonic epilepsy: underappreciated and under-diagnosed. Postgrad Med J 79: 78-80.

7. Arzimanoglou A, Guerrini R, Aicardi J (2004) Epilepsies with predominantly myoclonic seizures. Aicardis epilepsy in children. Philadelphia: Lippincott Williams \& Wilkins.

8. Hrachovy RA, Frost JD Jr (2006) The EEG in selected generalized seizures. $J$ Clin Neurophysiol 23: 312-332.

9. Marson AG, Al-Kharusi AM, Alwaidh M, Appleton R, Baker GA, et al. (2007) The SANAD study of effectiveness of valproate, lamotrigine, or topiramate for generalised and unclassifiable epilepsy: an unblinded randomised controlled trial. Lancet 369: 1016-1026. 